\title{
La resiliencia en la familia del niño con autismo: actitud y condición de desarrollo
}

\section{Resilience in the family of the child with autism: attitude and development condition}

\author{
M.Sc. Aylin Pentón Quintero ${ }^{1}$ \\ aylinpq@ucpejv.edu.cu \\ Dr. Rogelio Bermúdez Sarguera, PhD. ${ }^{2}$ \\ rogelio.bermudezs@ug.edu.ec, rbsarguera@gmail.com \\ Dra. Daisy de la Caridad Pérez Mato, PhD. ${ }^{3}$
}

Recibido: 1/04/2019; Aceptado: 1/06/2019

\begin{abstract}
RESUMEN
El presente artículo aborda la realidad inesperada que acarrea la presencia de un niño con autismo en la dinámica familiar, donde se requiere elevar los niveles de resiliencia para enfocar su desarrollo y detenimiento ante las adversidades. Se pretende concebir a la resiliencia más allá de una capacidad; pues con el intercambio y creación de redes de apoyo en la familia del niño con autismo, condición que ocurre como resultado de la consecución de un proceso en el cual intervienen distintos factores, ayudar a las familias a salir de dicha problemática de forma positiva para luego aprender de dicho acontecimiento o situación. El objetivo general es reflexionar acerca de la importancia que conlleva desarrollar la resiliencia en estas familias y círculo de relaciones, pues constituye actitud y condición innegable que les permita fortalecerse, de modo que los ubique en mejores condiciones de superarse a sí mismos y a su propio hijo. Mediante el uso de métodos investigativos como el análisis y síntesis y el histórico-lógico, se obtiene información de estudios que revelan la importancia del desarrollo de actitudes de resiliencia en las familias de personas con discapacida, y en este caso, de los niños con autismo.
\end{abstract}

Palabras Claves: autismo, familia, resiliencia

\footnotetext{
1 Universidad de Ciencias Pedagógicas "Enrique José Varona", Habana, Cuba.

2 Universidad de Guayaquil, Guayaquil, Ecuador.

3 Universidad de Guayaquil, Guayaquil, Ecuador.
}

Revista científica Ciencia y Tecnología Vol. 19 No 23 págs. 50-57

http://cienciaytecnologia.uteg.edu.ec 


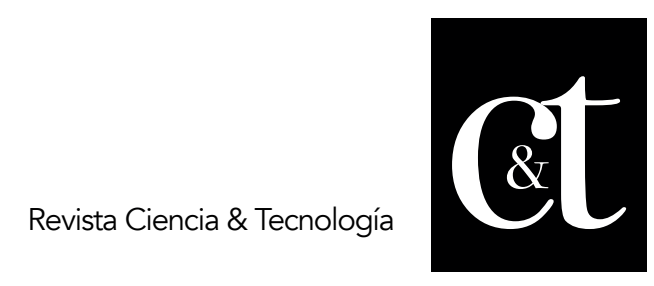

No. 23, 31 de julio de 2019

ISSN impreso: 1390 - 6321

ISSN online: 2661 - 6734

\begin{abstract}
This article focuses on autism: Attitude and developmental condition", addresses the unexpected reality of the presence of a child with autism in family dynamics, where raising levels of resilience to focus its development if it stops in the face of adversity. It is intended to conceive of resilience beyond a capacity, because with the exchange and creation of support networks, in the family of the child with autism occurs as a result of the achievement of a process in which different factors are involved that help to get out of this problematic positively and then learn from such an event or situation. It is intended, as a general objective, to reflect on the importance of developing resilience in these families and a circle of relationships, as it constitutes an undeniable attitude and condition that allows them to be strengthened, so that it places them in better conditions of overcoming themselves and their own child. Through the use of investigative methods such as analysis and synthesis and historical-logical, information is obtained from studies that reveal the importance of the development of resilient attitudes in families of people with disabilities, and in this case, children with disabilities Autism.
\end{abstract}

Keywords: autism, family, resilience

\title{
Introducción
}

La llegada de un nuevo miembro a la familia resulta un acontecimiento esperado por los miembros de ella y por otras personas cercanas a la dinámica familiar, entre ellos la propia comunidad. Ante un nuevo nacimiento todos se movilizan para dar la bienvenida al pequeño y enhorabuena a los padres que con gran motivo de alegría lo reciben y crean las condiciones para ello.

Resulta inevitable fantasear con la nueva presencia de un niño donde sin tener aún un rol activo, es depositario de expectativas y hechos por ocurrir en los deseos de los familiares. Pero no siempre la realidad ocurre de tal modo, en ocasiones surgen acontecimientos inesperados que deben ser afrontados y pueden generar estados emocionalmente lamentables; entre esos eventos adversos, se encuentra la llegada de un hijo con discapacidad.

Los padres se ven asediados por sentimientos que van desde el desconcierto, la ira y el resentimiento hasta la culpa, la impotencia y el arrepentimiento por reconocer lo que están sintiendo. La impotencia acompaña a los padres porque se desconoce con certeza etiología del trastorno o sobre lo que debe esperar de él. Se sienten muy solos porque no tienen un referente cercano acerca de alguien que haya padecido el síndrome del autismo y frustrados porque sus expectativas no se encontraban en correspondencia con el carácter irreversible del autismo.

El sufrimiento que dicha realidad les genera los acompaña por múltiples factores, entre ellos porque el amor de los hijos hacia ellos no se ha formado como en otros niños, típico de la propia naturaleza del autismo. Las intenciones de los padres para establecer relaciones afectivas con el niño con autismo a veces disminuyen, agudizándose las alteraciones en las relaciones interpersonales, muy difíciles de recuperar. 


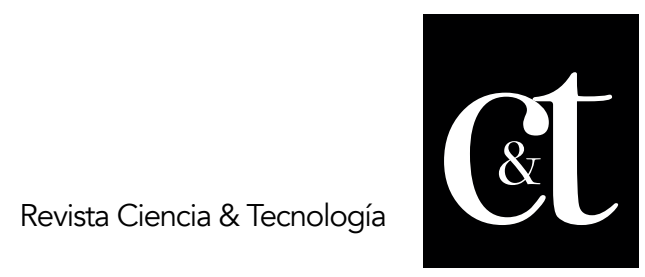

No. 23, 31 de julio de 2019

ISSN impreso: 1390 - 6321

ISSN online: 2661 - 6734

Aunque la sociedad continúa avanzando sin parar, continúan ideas erróneas en torno a las personas con discapacidad, de modo tal que el rechazo se encuentra visiblemente en el contexto social (Saad, 2010). Esto puede acarrear que las familias con hijos con discapacidad se vayan aislando cada vez más, reduciendo tanto el número de relaciones sociales y su calidad como las actividades de ocio.

La búsqueda de respuestas da comienzo de un largo camino. Es una etapa de esperanza e incertidumbre, la familia espera escuchar que la realidad de su hijo es una etapa de tránsito en su desarrollo, nada perenne ni trascendental. La intensa búsqueda de respuesta a la problemática que aqueja a la familia desata incertidumbres en cada uno de sus miembros donde afloran insatisfacciones, desvalorización en su rol y vivencia de soledad. Los estados emocionales negativos desarman a la familia de una actitud resiliente y pueden llegar a comportamientos reproductores del aislamiento, alejándolos de asumir enteramente su función educativa y gestión socializadora.

La familia ha aprendido que hay ciertas estrategias que pueden usar para tolerar mejor el impacto emocional, físico e intelectual que el autismo trae consigo, y solo desarrollándose en ellos la cualidad para adecuarse de forma positiva a situaciones emocionalmente difíciles, lograrán sobreponerse a dichas adversidades y continuar la vida con mayor fortalecimiento, a ella, le llamamos "resiliencia".

La resiliencia es un concepto que conquista posiciones en Psicología y es un valor en alza en los nuevos planteamientos y actitudes psicológicas. Debemos dirigirnos a la obra de John Bowlby para hallar las primeras referencias al término de resiliencia, quien lo definiría como la capacidad de los seres humanos para superar etapas de dolor emocional y situaciones adversarias, saliendo fortalecido de ellas.

\section{Desarrollo}

El proceso educativo desde la familia con un niño con autismo se caracteriza por ser triste, angustioso, confuso, ansioso, ya que durante los primeros meses de vida del niño todo transcurre dentro de lo esperado y hasta precoz en algunas ocasiones, y se respira felicidad en cuanto a la relación familia-hijo. De forma paulatina aparecen síntomas de interés que detonan gran diferencia en el desarrollo del niño.

El proceso de diagnóstico es extenso, tenso, generando limitaciones sociales, pues se les hace difícil la convivencia en lugares públicos, por lo que se manifiestan intranquilas, inseguras y muy temerosas.

Cuando el niño con autismo es el primer hijo, esta situación es más difícil de identificar, pues suelen carecer de experiencias sobre los indicadores de desarrollo en las edades tempranas y resulta complejo darse cuenta de la existencia de una alteración. Por lo general, suelen demorar más en acudir al especialista, en ocasiones solicitan atención médica porque otros miembros de la familia se percatan de la existencia de rasgos "extraños", o quizás alguna persona se le acerca, para llamarles la atención sobre el desarrollo de su hijo. Debido a estas alarmas, comienzan a sospechar que algo anda mal, comienza la comparación con otros niños, se vuelven más observadores, valoran que el desarrollo de su hijo es diferente, y socorren a la ayuda profesional al encuentro de explicación, de un "diagnóstico certero". 


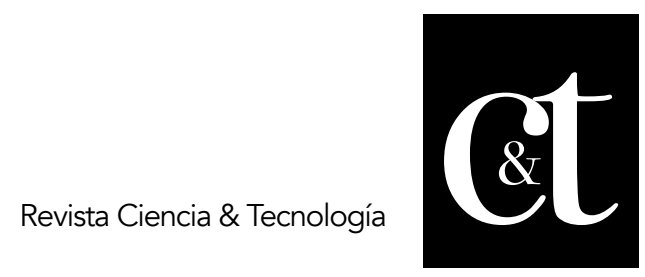

No. 23, 31 de julio de 2019

ISSN impreso: 1390 - 6321

ISSN online: 2661 - 6734

La confirmación del diagnóstico de autismo es un fuerte impacto que implica una reacción en todos y cada uno de los miembros de la familia. Esta etapa es descrita con diferentes términos: impacto, choque emocional, shock. Ese estado, se produce desde el momento en que se recibe la noticia y tiene la vivencia directa de que el niño no es como los otros, pero no lo acepta como real, los padres presentan una actitud que pudiera parecer de apatía, discrepancia, y muestran expresiones llenas de dolor y desconcierto. Pues aun cuando sus sospechas se acercaban al diagnóstico recibido, la negación del mismo como mecanismo de defensa persiste y la respuesta a su realidad los alarma de un modo devastador. La familia, en especial los padres, no se encuentran preparados para escuchar su hijo, aquel que con tantas ilusiones esperaban, que parecía "normal" hasta un momento dado de su desarrollo, padece un trastorno grave y que resulta irreversible.

La familia del niño con autismo difícilmente encuentra consuelo en la sociedad, encuentran un mundo insensible, despiadado y hostil. La percepción de incomprensión ante las conductas extrañas del niño, provoca en los padres sentimientos de aislamiento. Suelen sentirse avergonzados por no haber cumplido con las expectativas sociales de tributar un ciudadano fundamentalmente productivo.

Se sienten excluidos de situaciones normales de ésta, debido a que vivimos en una época en que se exige el progreso donde el desarrollo es básico y la excelencia y la calidad son las palabras que norman el quehacer cotidiano.

Cuando la familia ha canalizado los sentimientos antes mencionados, logran aprovechar sus potencialidades y estimular el desarrollo de habilidades en su hijo, experimentan la aceptación del niño por encima del trastorno y así el propio niño le devuelve al ambiente familiar seguridad, empeño y unidad entre sus miembros. Existe un diverso número de canales que han favorecido la supervivencia de una familia con una persona con autismo, lo que demuestra que es posible, aunque difícil.

Desde la época de los 60 han sido muchos los cambios que este término ha sufrido hasta la época actual. Inicialmente se consideraba a la resiliencia como una condición natural, innata, de los humanos, más tarde fueron incorporados elementos culturales, familiares, comunitarios y sociales, pues según investigaciones al respecto, éste es un proceso social en donde influyen en gran medida los aspectos del entorno que rodea al sujeto. Uno de los criterios mejores defendidos, al no referirse únicamente como capacidad o cualidad, es el ofrecido por Santos (2000), donde asegura que la Resiliencia es saber afrontar la adversidad de forma constructiva. Saber adaptarse con flexibilidad y salir fortalecido del suceso traumático, según Santos (2000).

Ante un momento difícil emocionalmente hablando, la familia, los amigos y el entorno en general, son elementos que influyen en gran medida, es por ello que no se puede ver a la resiliencia como una capacidad únicamente, debido a que más bien es la consecución de un proceso en el cual intervienen distintos factores que ayudan a salir de dicha problemática de forma positiva para luego aprender de dicho acontecimiento o situación. En el caso de la familia del niño con autismo, el acontecimiento adverso no se limita al propio momento de desconcierto que genera el impacto del diagnóstico 


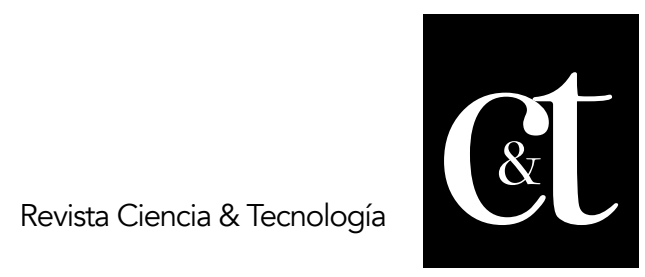

No. 23, 31 de julio de 2019

ISSN impreso: 1390 - 6321

ISSN online: 2661 - 6734

sino que trasciende a representarse una ruptura en el ideario y expectativas para con el pequeño niño que llevará consigo durante toda la vida una condición singular. Por lo tanto, la resiliencia en estas familias y círculo de relaciones, resulta ser una perenne actitud, vivir fortaleciéndose para encontrarse en mejores condiciones se superarse a sí mismos y a su propio hijo.

En este sentido, no solo podemos analizar su papel individual de cada uno de los padres, sino también, su entorno familiar y social. La familia tiene huella en nuestra vida desde nuestra niñez, es en ella donde se concretan las primeras relaciones sociales. A través de la resiliencia familiar es posible desarrollar recursos con los que enfrentar una crisis, pues estas son un factor a tener en cuenta en los ciclos familiares. La familia es un sistema que tiene sus propias estructuras y dinámicas de comportamiento.

Las características propias del contexto familiar hacen que cada familia tenga un desarrollo peculiar. Si logran tener una actitud resiliente ante esta situación atormentadora, seguramente será más viable el manejo de esas circunstancias delicadas cuando interactúan con personas ajenas a la convivencia familiar en diferentes contextos sociales.

Gómez, I. (2005), alega que para que la familia logre aceptar este diagnóstico es necesario que:

- Domine mecanismos de canalización de los sentimientos negativos y los transformen en fuerza que la impulse a aprovechar el potencial del niño para su educación y desarrollo.

- Concientice que se tiene un niño, no un enfermo; se evite el proteccionismo extremo que influye negativamente en las posibilidades de desarrollo y compensación.

- Comprenda que los trastornos autistas pueden limitar el aprendizaje, pero que estos niños son capaces de desarrollar determinadas habilidades cognitivas, afectivas, comunicativas y sociales.

- Esté dispuesta a reorganizar los roles de las figuras parentales en el contexto familiar y social, a realizar cambios en el hogar (incluyendo los físicos), para que el niño no se exponga a peligros que puedan prevenirse.

- Tenga presente que la presencia del niño no significa para los padres el abandono de sí mismos, sino que estos deben satisfacer, en su condición de seres humanos, sus necesidades materiales y espirituales.

- Cree un ambiente de seguridad, aceptación, respeto, para que el niño pueda desarrollar las habilidades que le permitan integrarse y actuar en los diferentes contextos, de manera que la familia pueda crecer, convivir, establecer relaciones satisfactorias y gratificantes.

En términos de resiliencia en las familias de los niños con autismo, se debe partir del autoconocimiento de capacidades y vulnerabilidad de cada persona y grupo familiar. Dichos resultados favorecen poner en marcha un programa determinado centrado en las áreas más vulnerables para fortalecer a la persona y con ellos a su familia. Santos (2000) propone diez pilares en esta dirección:

1. Introspección: capacidad de observarse, de conocerse a sí mismo y darse una 


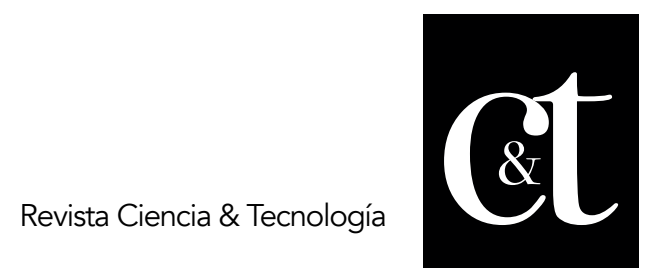

No. 23, 31 de julio de 2019

ISSN impreso: 1390 - 6321

ISSN online: 2661 - 6734

respuesta honesta en relación al mundo exterior.

2. Motivación esencial: capacidad de darle sentido a la vida creando su propio proyecto transcendente.

3. Autorregulación emocional: capacidad de afrontar tensiones sin victimismo como parte de la vida, debilitando la respuesta al estrés.

4. Independencia y autonomía emocional: capacidad de mantener distancia emocional y física ante los conflictos sin caer en el aislamiento. Saber fijar límites entre uno mismo y el medio con problemas.

5. Confianza en sí mismo y en sus propios recursos: adecuada autoestima, iniciativa y responsabilidad para lograr autonomía personal.

6. Capacidad de relacionarse: habilidad para establecer vínculos afectivos con otras personas creando relaciones saludables. Equilibrar la propia necesidad de afecto con la actitud de ayudar a otros.

7. Actitud positiva y optimismo: capacidad para resolver problemas de forma creativa, desdramatizando.

8. Sentido del humor y creatividad: para resolver problemas relativizando y sabiendo encontrar lo cómico en la propia tragedia.

9. Colaboración y compromiso: capacidad de comprometerse con valores y ayudar a otros.

10. Moralidad, ética y coherencia: mantener una unidad de vida.

El trabajo con las familias, en especial preparar a las familias de los niños con autismo, para que se encuentren en mejores condiciones de educar a sus hijos y a sí mismos en su realidad, no consiste en fácil labor para profesionales de la psicología y pedagogía. Se debe acudir a redes de apoyos que fortalezcan los contextos donde se hace más vulnerable su propio desarrollo, donde conviven a diario y enfrentan dificultades de comprensión, tolerancia, respecto, apoyo y acompañamiento emocional.

\section{Conclusiones}

Resulta imprescindible generar un ambiente que propicie buenas relaciones interpersonales en una comunidad, para que permita la inclusión social de las personas con discapacidades teniendo muy claro, que tanto el respeto como la solidaridad son dos valores imprescindibles para que sea posible convivir armónica desde la diferencia. Las áreas en las que se trabaja para superar los conflictos son:

La adaptación o reformulación de la vida tras el conflicto

La búsqueda de un sentido o propósito en la vida

La construcción de una red social de apoyo 


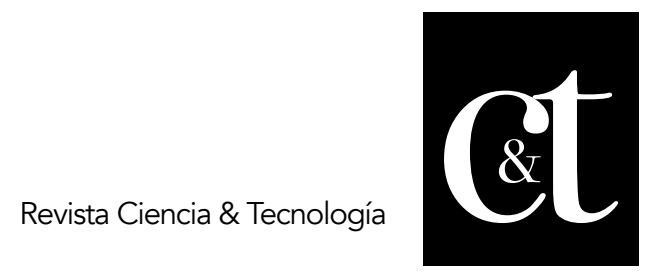

No. 23, 31 de julio de 2019

ISSN impreso: 1390 - 6321

ISSN online: 2661 - 6734

Acciones educativas intencionadas conjuntamente con los docentes, la familia y la comunidad, que garanticen mejores condiciones de enfrentar la problemática del autismo en la sociedad

En la realidad que acoge al autismo, es recurrente la idea de que la sociedad ya no ve a la familia como antes, sino que los consideran como personas extraordinarias que tienen que ayudar a su hijo "con problemas" (Novell, 2010). Será importante que recuerden que a pesar de que hacen esfuerzos permanentes para ayudar a su hijo, continúan siendo personas aun con más necesidad de recobrar su autoestima, capacidad de trabajo y de disfrutar de las cosas positivas de su proyecto de vida.

Se puede evidenciar como característica recurrente y propia en la familia del niño con autismo, que lejos de la ruptura emocional que suele disolver la unión matrimonial consecuencia de la culpa y rechazo sentido, tienden a unirse y cuentan además con el apoyo de otros familiares, fundamentalmente convivientes. Esta integración que experimentan los padres es muestra de crecimiento alcanzado una vez aceptada la situación y en proceso de elaboración conjunta, donde los sitúa entonces en nuevas y buenas condiciones de cumplir con una de las funciones primordiales de la familia, la función educativa.

No se debe tomar pasivamente la realidad discriminatoria que aqueja a los "más diferentes", es necesario que sean las potencialidades humanas las que denoten las diferencias, accionando también sobre esas necesidades en su variado espectro y extrayendo asimismo de las minorías sus posibilidades, aunque puedan parecer poco significativas. La comunidad debe ser vista como uno de los espacios propicios para el rescate y desarrollo de las fortalezas de personas con marcadas dificultades en el área de la socialización fundamentalmente.

\section{Referencias bibliográficas}

Campo, I. (2012). "Una estrategia de educación familiar en la escuela para niños con autismo". Tesis doctoral. La Habana.

Cañedo, G. (2005) "La Familia cubana y la Cultura de la Diversidad".

Pedagogía curso 56. IPLAC.

Castro, P. L. (2002). "Reflexiones y experiencias con los padres en Educación Especial". Convocados por la diversidad.

Novell, R., Rueda,P. y Carrillo, L. (2010). Salud mental y alteraciones de la conducta de las personas con discapacidad intelectual. Cádiz, España: FEAPS.

Pentón, A. (2017) "Estrategia de Educación familiar en la comunidad vecinal donde residen niños con autismo". ICCP. Centro de Referencia Latinoamericano para la Educación Especial. Tesis de Maestría.

Saad, E. et al. (2010). Salud mental infanto juvenil: prioridad de la humanidad. 3ra. Ed. Perú; Ediciones científicas APAL 


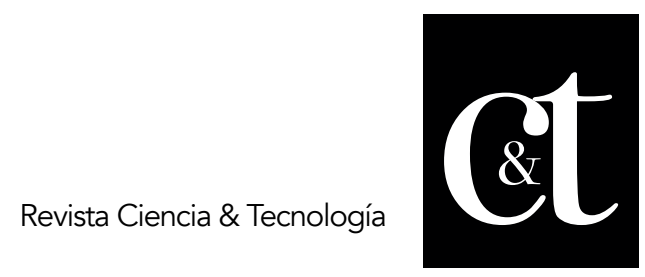

No. 23, 31 de julio de 2019

ISSN impreso: 1390 - 6321

ISSN online: 2661 - 6734

Santos, R. (2009). "Desarrollo de la personalidad y resiliencia". Ilustre Colegio de Médicos de Madrid Congreso SETEPT 2009. Ponente.

Uriarte, A. (2013) "La perspectiva comunitaria de la resiliencia". Universidad del País Vasco Psicología Política, No 47. 